\title{
Körpersprachen der Unsagbarkeit
}

Leibliche Grenzerfahrungen in literarischen Texten über die Shoah

Langages corporels de l'indicible. Expériences-limites corporelles dans des textes littéraires sur la Shoah

Body Languages of the Unutterable. Boundary Experiences in Shoah Literature

\section{Christiane Solte Gresser}

\section{(2) OpenEdition Journals}

Édition électronique

URL : https://journals.openedition.org/ceg/9741

DOI : $10.4000 /$ ceg. 9741

ISSN : 2605-8359

Éditeur

Presses Universitaires de Provence

Édition imprimée

Date de publication : 20 janvier 2020

Pagination : 111-125

ISBN : 979-10-320-0253-7

ISSN : 0751-4239

Référence électronique

Christiane Solte Gresser, „Körpersprachen der Unsagbarkeit“, Cahiers d'Études Germaniques [Online], 78 | 2020, Online erschienen am: 28 Oktober 2021, abgerufen am 02 Februar 2022. URL: http:// journals.openedition.org/ceg/9741 ; DOI: https://doi.org/10.4000/ceg.9741 


\title{
Körpersprachen der Unsagbarkeit Leibliche Grenzerfahrungen in literarischen Texten über die Shoah
}

\author{
Christiane SOLTE-GRESSER
}

Universität des Saarlandes, Saarbrücken

An einem „Wörterdelirium“ zu ersticken; sprechen zu müssen, ohne sprechen zu können; das ist die Aporie, die Sarah Kofman in Paroles suffoquées beschreibt. ${ }^{1}$ In diesem Essay versucht sie, wie so viele andere ${ }^{2}$, eine Sprache für die Erfahrungen der Shoah zu finden. In der Auseinandersetzung mit Robert Antelmes L'Espèce humaine $^{3}$, den Schriften von Maurice Blanchot ${ }^{4}$ und nicht zuletzt mit ihrer eigenen Geschichte als Holocaust-Überlebende ${ }^{5}$ denkt sie über die „physische Unmöglichkeit zu sprechen“ nach; über Worte, die einem den Atem nehmen und die Möglichkeit verhindern, zum Sprechen anzusetzen. ${ }^{6}$ Das „Unaufhörlichsprechen-Müssen“, das sich mit „unwiderstehlicher Gewalt aufdrängt“, steht einem „Ersticktwerden“ durch die erlittenen Erfahrungen unvermittelbar gegenüber. Diese Sprachlosigkeit fasst Kofman mit Blanchot in das Bild eines „Schweigens - wie ein Schrei ohne Worte, stumm, aber unaufhörlich schreiend“.7

1. Sarah Kofman, Paroles suffoquées, Paris, Galilée, 1987; dt.: Erstickte Worte, aus dem Französischen von Birgit Wagner, Wien, Passagen, 2005, S. 51.

2. Vgl. etwa James E. Young, Beschreiben des Holocaust. Darstellung und Folgen der Interpretation, Frankfurt a. M., Suhrkamp, 1997; Lawrence L. Langer, Holocaust Testimonies: The Ruins of Memory, New Haven, Yale University, 1991, um nur zwei repräsentative Ansätze von theoretischer Seite zu nennen. Neben Antelme ließe sich auf literarisch-autobiographischer Seite vor allem das Schreiben von Primo Levi und Jorge Semprún als besonders bedeutsam aufführen, zumal diese drei Autoren - für das Thema des vorliegenden Aufsatzes entscheidend - Träume verwenden, um die Erfahrung zwischen alptraumhafter Realität, traumhafter Entfremdung, Sprachdrang und Sprachversagen auszuloten.

3. Robert Antelme, L'Espèce humaine, Paris, Gallimard, 2014 [1957] (zur Thematik des NichtSprechenkönnens als körperliche Erfahrung vgl. unter anderem S. 100).

4. Maurice Blanchot, Le Pas au-delà, Paris, Gallimard, 1973; La Folie du jour, Paris, Fata Morgana, 1973 (dt. Köln, 1986) und vor allem „L'Idylle“, in Maurice Blanchot, Après coup, Paris, Minuit, 1983.

5. „Mon père est mort à Auschwitz. Comment ne pas le dire? Et comment le dire?“, siehe den Klappentext von Kofman, Paroles suffoquées.

6. Kofmann, Erstickte Worte, S. 51.

7. Ibid., S. 26 angesichts der neutralen, pathos-losen Gedächtnisschrift von Serge Klarsfeld (von der ein Ausschnitt in Paroles suffoquées eingefügt ist) und in Auseinandersetzung mit einem Blanchot-Zitat aus Blanchot, Le Pas au-delà, S. 87. 
Für Kofman ist es unhaltbar, über die Shoah in Form einer klassischen Erzählung zu kommunizieren. ${ }^{8}$ Die Grenzerfahrung unendlicher Bedürftigkeit, sagt sie, kann nicht mitgeteilt werden, ohne zu schwindeln. Gleichwohl existieren zahlreiche Versuche, eben eine solche Unmöglichkeit des Sprechens erzählend zu inszenieren - nicht nur in autobiographischen Zeugnissen, sondern auch im fiktionalen Roman; eine Ausdrucksform, die auch Shoah-Überlebende selbst durchaus sehr bewusst verwenden, wie dieser Beitrag zeigen wird. ${ }^{9}$ Solche fiktionalen Auseinandersetzungen erfolgen keineswegs unreflektiert ${ }^{10}$; ihre Autoren wissen, dass sie sich damit in eine komplexe Kontroverse hineinbegeben, indem sie sich etwa dem Vorwurf der Frivolität vor allem seitens jüdischer Überlebender aussetzen. ${ }^{11}$ Was diese Texte genre-unabhängig gemeinsam haben, ist ein Ringen mit Worten, das sich stets zwischen den Extremen eines völligen Verstummens auf der einen und geschwätziger Trivialisierung auf der anderen Seite bewegt. Wie sich das ,Unaussprechliche' in einzelnen literarischen Texten über die Shoah als körperliche Erfahrung niederschlägt, und wie ein solcher Zustand literarisch anhand der Grenzen des menschlichen Körpers gestaltet wird - nämlich durch die Konzentration auf bestimmte Körperorgane auf dem schmalen Grat zwischen Leben und Tod -, ist das Thema dieses Beitrags. Erörtern

8. Kofmann, Erstickte Worte, S. 30; vgl. auch ibid., S. 51: „Wie soll man also erzählen, was nicht ohne Schwindel ,mitgeteilt‘ werden kann? Das, wofür es zu wenig - oder zu viele - Worte gibt, um es zu sagen, und nicht nur, weil die „Grenzerfahrung“ der unendlichen Bedürftigkeit [wie jede andere Erfahrung] nicht vermittelt werden kann?“

9. Vgl. die Werke von Anna Langfus und Edgar Hilsenrath in diesem Beitrag. Zu ergänzen bleibt, dass auch eine historische bzw. historiographische Perspektive sich des Repräsentationsproblems sehr bewusst ist. Saul Friedlander etwa verbindet mit seinen Forschungen zur Judenverfolgung und Judenvernichtung die unlösbare Aufgabe, den Ermordeten eine Stimme zu verleihen, was ihmzufolge auch die Geschichtswissenschaft an ihre eigenen Grenzen führe. Interessanterweise macht auch er dieses Problem an der Stimme bzw. am nicht mehr hörbaren Schrei der Opfer fest: Gerade die „unerwarteten Schreie und geflüsterten Worte“, die „Unmittelbarkeit des Schreies eines Zeugen“ seien es, die die "gut geschützte“ emotionale Distanz des Historikers und seiner Öffentlichkeit erschüttern (vgl. Saul Friedlander, Das Dritte Reich und die Juden. Die Jahre der Vernichtung 1933-1945, Bd. 2, München, Beck, 2006, S. 24-25). Zum Repräsentationsproblem insgesamt vgl. Saul Friedlander (Hrsg.), Probing the Limits of Representation. Nazism and the ,Final Solution', Cambridge, Havard UP, 1992.

10. Vgl. um einmal nur die ästhetisch-poetologischen Positionen der AutorInnen dieses Beitrags zu nennen: Anna Langfus: „Un cri ne s'imprime pas“, in Clara Lévy, „La guerre dans les textes littéraires d'Anna Langfus: la mise à distance de l'expérience“, Esprit Créateur 40/2, 2000, S. 52-60, hier S. 59; Charlotte Delbo, La Mémoire et les Jours, Paris, Berg International, 2013; Marcel Beyer, „Ich glaube, es ist leichter, wenn man glaubt“, in Martin Doerry (Hrsg.), Nirgendwo und überall zu Haus. Gespräche mit Überlebenden des Holocaust, München, DVA, 2006, S. 50-59; Edgar Hilsenrath, „Deutsch war nicht die Sprache der Nazis. Es war meine Sprache“, Wiesbaden, Aktives Museum Spiegelgasse für Deutsch-Jüdische Geschichte, 2009, 2 CDs. Vgl. dazu insgesamt auch die Ausgabe Literatur und Holocaust in Text und Kritik 144, 1999, in der Marcel Beyer auf die Frage, ob man über den Holocaust schreiben darf, wenn man ihn nicht erlebt hat, mit einem dezidierten Ja antwortet, seine Antwort sodann jedoch sorgsam differenziert und reflektiert (ibid., S. 18-24).

11. Dass es sich hierbei nicht nur um eine Kontroverse zwischen biographischer Nähe oder Distanz zur Shoah handelt, sondern auch um einen Unterschied zwischen literarischen und philosophischen Positionen, betont Jürg Altwegg, „Schreiben nach - und über - Auschwitz. Vorwort zur ersten deutschen Ausgabe“, in Kofmann, Erstickte Worte, S. 12-22, hier S. 21. 
möchte ich es anhand einer Textauswahl, die ausdrücklich fiktionale Erzählungen und autobiographische Zeugnisse mit einbezieht und ein gemeinsames Kriterium besonders berücksichtigt: In sämtlichen Texten stellen Träume einen entscheidenden Wahrnehmungsmodus und das vorherrschende Erzählverfahren dar, um sich mit dem Problem der Sprachlosigkeit auseinanderzusetzen.

Edgar Hilsenraths Roman Der Nazi \&t der Friseur von 1971/1977 erzählt die Geschichte des SS-Massenmörders Max Schulz. Dieser nimmt nach dem Krieg die Identität seines jüdischen Jugendfreundes an, den er als KZ-Aufseher selbst erschossen hatte. Eine Episode dieser bösen Satire handelt von der wundersamen Verwandlung der KZ-Überlebenden Mira, die den NS-Verbrecher unwissend heiratet und ihr Trauma von einem Moment zum nächsten überwindet: Seit sie bei einer missglückten Erschießung durch die SS (an der der Erzähler-Ehemann womöglich selbst beteiligt war) wieder „aus dem Massengrab herausgekrabbelt“ 12 ist, hat Mira ihre Stimme verloren. Nachdem sie auch das Konzentrationslager - bis auf die Knochen abgemagert - überlebt, verbringt sie die Nachkriegsjahre in Palästina, gänzlich ohne zu sprechen; als „eine stumme Freßmaschine“, wie der Erzähler in der für ihn typischen Derbheit feststellt. Einen Höhepunkt dieser wahnwitzigen Überkreuzung von Täter- und Opfergeschichte bildet der Moment, in dem Mira ihre Sprache wiederfindet: Zum Essen geladen, bekommt das Paar - ausgerechnet in Israel und ausgerechnet am Sabbatnachmittag deutschen Sauerbraten mit Klößen serviert. Die grotesk überspitzte ,Heilung“ der KZ-Überlebenden gestaltet sich als nahtloser Übergang vom sprachlosen Insichhineinfressen zum Wiedererlangen der eigenen Stimme:

[Mira hob] beunruhigt den massigen Kopf. Ich konnte sehen: ein dicker deutscher Kloß steckte in ihrem Mund. Und sie konnte ihn weder hinunterschlucken noch ausspucken. [...] Ich habe Mira noch nie weinen sehen. [...] Und ich sah: das Starre in ihren Augen wurde flüssig, zerschmolz, rann aus ihren Augen heraus, floß aus dem Seelenspiegel, über Wangen und Kinn, tropfte auf den zerfallenen Kloß [...]. Mira wollte laut weinen. Ihr Mund war sperrweit offen. Aber keine Laute kamen aus diesem Mund. Gewiß, dachte ich, steckten dort viele Klöße! [...] Ich dachte: die unsichtbaren Klöße. Das sind die richtigen Klöße. [...] Mira zitterte am ganzen Körper. [...] Weinte mit offenem Mund. Und spuckte ihre Klöße aus. [...] Wir zuckten plötzlich alle zusammen... denn Mira hatte einen Schrei ausgestoßen... und dann... auf einmal... begann Mira, die Stumme, zu reden. ${ }^{13}$

Auch ein anderer Holocaust-Roman ${ }^{14}$ mit nachweisbaren Bezügen zu tatsächlichen Tätern des Nationalsozialismus ${ }^{15}$, lotet den Zusammenhang von Sprache und Sprachlosigkeit aus; genauer: die Grenzen des Körpers zwischen Sprachmacht,

12. Edgar Hilsenrath, Der Nazi \&t der Friseur. Gesammelte Werke, Bd. 2, Berlin, Dittrich, 2004 [1977], S. 379.

13. Ibid., S. 402-403.

14. Vgl. Ulrich Simon, „Assoziation und Authentizität. Warum Marcel Beyers Flughunde auch ein Holocaust-Roman ist“, in Marc-Boris Rode, Auskünfte von und über Marcel Beyer, Bamberg, Universität Bamberg, Reihe: Fußnoten zur Literatur, 2003, S. 126-145.

15. Vgl. hierzu ausführlicher Philipp Alexander Ostrowicz, Die Poetik des Möglichen. Das Verhältnis von ,historischer Realität ' und ,literarischer Wirklichkeit' in Marcel Beyers Roman ,Flughunde", Stuttgart, ibidem, 2005. Zu historischen Referenzen bei Hilsenrath vgl. Susann Möller, Wo die Opfer zu Tätern werden, machen sich die Täter zu Opfern: Die Rezeption der beiden ersten Romane Edgar Hilsenraths in Deutschland und in den U. S. A., Ann Arbor/ Michigan, UMI, 1991, 
Stimmgewalt, Verschweigen, Versagen und Verstummen. Marcel Beyers Flughunde von 1995 erzählt ebenfalls aus der Täterperspektive, und die Körper-Grenzen der NS-Opfer bilden hier sogar den entscheidenden Erzählfokus. So können folgende Eingangsüberlegungen des Protagonisten als programmatisch für den gesamten Roman gelten:

Geformter Atem, Hauch: Das, was den Menschen ausmacht. So bilden die Narben auf den Stimmbändern ein Verzeichnis der einschneidenden Erlebnisse, akustischer Ausbrüche, aber auch des Schweigens. Wenn man sie nur mit dem Finger abtasten könnte, mit ihren Fährten, Haltepunkten, Verzweigungen. Dort, in der Dunkelheit des Kehlkopfs: Das ist deine eigene Geschichte, die du nicht entziffern kannst. [...] Wenn vom einen auf den anderen Moment der Mund austrocknet, wenn sich der Hals zuschnürt, wenn dich, scheinbar grundlos, Atemnot befällt und aus den Lungen nur das eine dringt: nichts. ${ }^{16}$

Hermann Karnau, als Akustiker für Propagandaminister Goebbels tätig, experimentiert zunächst eher zufällig mit der menschlichen Stimme und ihren technischen Aufzeichnungsmöglichkeiten ${ }^{17}$, bevor er schließlich zum (Pseudo-)Wissenschaftler ${ }^{18}$ wird, der umfangreiche medizinische Experimente am Stimmapparat von KZ-Häftlingen durchführt. Sein Ziel ist eine Kartierung sämtlicher Nuancen menschlicher Laute und stimmlicher Klangfarben. $\mathrm{Zu}$ diesem Zweck ,testet‘ er, wie belastbar die Sprechwerkzeuge seiner Gefangenen sind: Größte Aufmerksamkeit wird hier einzelnen Organen gewidmet, die mit der menschlichen Artikulationsfähigkeit verbunden sind; neben dem Kehlkopf etwa Zunge, Stimmbändern, Rachen und Luftröhre. Das Spektrum der akustischen Wahrnehmungen auf der Grenze zwischen Leben und Tod wird von Marcel Beyer mit brutaler Kunstfertigkeit vorgeführt:

Nur noch animalische Töne, sie werden jetzt nicht mehr geformt im Kehlkopf, und werden nicht gedämpft im Hals, sie erfüllen den ganzen Rachenraum. Und Lippen, Zunge, Zähne können diese ungewollten Laute auch nicht mehr im Zaum halten, aufhalten und zum Verstummen bringen noch im Mund. Welch ein Geschehen. Welch ein Panorama. ${ }^{19}$

und Astrid Klocke, The Concept of Black Humor and Edgar Hilsenrath's Novel ,Der Nazi und der Friseur;, Ann Arbor/ Michigan, UMI, 2001.

16. Marcel Beyer, Flughunde, Frankfurt a. M., Suhrkamp, 2012 [1995], S. 22-23. Mit dem Zusammenhang zwischen Sprechen, Sprechverbot und Sprachzwang im Nationalsozialismus setzt sich Marcel Beyer in seiner Lektüre des Leiris’schen Essays „Was Sprechen heißt“ auseinander, den er als eine der wenigen poetologischen Vorgaben für seine eigene Arbeit bezeichnet (Beyer, „Spucke“, Anhang in Flughunde, S. 271-284, hier S. 272) und S. 282: „Der Körper ist nicht Gedächtnis, sondern er hat Gedächtnis: Mit dieser Vorstellung von Körpergedächtnis gelingt es mir nicht mehr, Geschichte als einen von mir abgetrennten Bereich zu begreifen, aus dem ich mich ausklammern könnte, zu dem ich mich in ein Verhältnis setze“. Vgl. hierzu auch das Nachwort von Marcel Beyer, Flughunde, S. 285-294, bes. S. 290-291.

17. So konstatiert er beim Festhalten der letzten Laute von fallenden Soldaten an der Front: „da hat es einen jungen Sterbenden richtig herausgehauen aus der Lautbeherrschung“ (Beyer, Flughunde, S. 111).

18. Vgl. hierzu Roman Pliske, „Flughunde. Ein Roman über Wissenschaft und Wahnsinn ohne Genie im ,Dritten Reich““, in Marc-Boris Rode (Hrsg.), Auskünfte von und über Marcel Beyer, S. 110125.

19. Beyer, Flughunde, S. 106. 
Solchen Körperbeschreibungen zwischen Sprachvermögen und Sprachzerstörung möchte ich nun weiter nachspüren, indem ich mich von der literarisch inszenierten Täterperspektive zu den körperlichen Erfahrungsdimensionen der Opfer hinbewege. Es geht mir zum einen um die Frage, wie diese in den Beispielen literarisch inszeniert werden. Zum anderen interessiert mich, wie die individuell und subjektiv wahrgenommenen Sprachorgane erzählerisch mit dem allgemeinen Topos der Unsagbarkeit verbunden sind. In diesem Zusammenhang verdient eine auffällige Gemeinsamkeit der ausgewählten Shoah-Texte besondere Aufmerksamkeit: Sie alle verwenden Traumdarstellungen, Traumepisoden oder ein traumhaftes Schreiben; genauer: Sie erzählen von Träumen, in denen die subjektiven Erfahrungen von Körpergrenzen besonders eindrücklich in Szene gesetzt werden. Gerade im Traumerleben werden, so wird sich zeigen, vielfache Grenzen überschritten: Die Grenzen zwischen Tätern und Opfern, die Grenzen zwischen Leben und Tod, die Grenzen der eigenen Körpererfahrung, aber auch räumliche und zeitliche Grenzen, mit denen Erinnerungen und Erfahrungen der Shoah den Träumenden auf den Leib rücken.

Für die skizzierte Fragestellung ist die erzähltechnische Gestaltung von Beyers Roman in mehrfacher Hinsicht bedeutsam: Der Zusammenhang von Körper, Stimme und Ausdrucksfähigkeit wird hier aus der kalten, teilnahmslosen, um Objektivität bemühten Täterperspektive ausgelotet. ${ }^{20}$ Die Misshandlungen sind in erster Linie von außen, an den physischen Reaktionen der Opfer ablesbar; als (pseudo-)wissenschaftliches Phänomen sind diese Gegenstand akustischer Aufzeichnungstechniken. ${ }^{21}$ Über die gewaltsame Zerstörung ihrer Sprechorgane hinaus, werden die Opfer als menschliche Individuen also auch narrativ ausgelöscht: Sie sind namenlos, gesichtslos, geschichtslos; hinter dem biologischen ,Material' für medizinische Experimente werden für den Täter - und folglich auch für uns LeserInnen ${ }^{22}$ - lediglich schwarze Schatten, „Schemen“ ${ }^{23}$ sichtbar, die weder selbst die Stimme ergreifen und für sich oder die

20. Die andere Stimme, die sich mit der ,Täterperspektive“ abwechselt, ist diejenige der ältesten Goebbels-Tochter, die zusammen mit ihren Geschwistern in Karnaus Obhut gegeben wird.

21. „Schallquelle (vgl. auch „Resonanzkörper“, S. 61), nicht etwa Mensch mit Schmerzen [...]. Ich darf mich von einer bemerkenswerten Stimme nicht ablenken lassen durch das viehische Gebaren [...]. Vielleicht macht ein bestimmtes Wimmern Vergleichsaufnahmen nötig, um die Nuancen dieses Klagelauts im Kartenwerk zu erfassen“ (Beyer, Flughunde, S. 30).

22. Hierin ist Beyer ausgesprochen konsequent. Es geht ihm um Reflexion, nicht um Empathie mit den Opfern, die er selbst für unzureichend hält. Denn er hat den berechtigten Verdacht, „es geh[e] uns, indem wir das historische Material vom imaginären trennen, weniger um Erkenntnis als darum, Souverän über den Bereich unserer Gefühle zu bleiben“. „Jeder einzelne Satz in „Flughunde“ sollte zu erkennen geben, daß er nicht aus eigenem Erleben formuliert worden ist, ein Werk der Imagination, nicht der Erinnerung. Dies war meine Haltung denjenigen gegenüber, die sich erinnern“; alle Zitate in Beyers Nachwort, ibid., S. 289.

23. Beyer, Flughunde, S. 11. Zu derartigen Begriffen, die auf das Phänomen des „Muselmanns“ im Lager zielen, vgl. Christiane Solte-Gresser, „Das Sterben der Anderen. Zur Traum-Sprache des ,Muselmanns‘ bei Delbo und Vercors, in Mauro Fosco Bertola, Christiane Solte-Gresser (Hrsg.), An den Rändern des Lebens. Träume vom Sterben und Geborenwerden in den Künsten, Paderborn, Fink, 2019, S. 263-284. 
Mitgefangenen zeugen können, noch für die jemand anderes zu zeugen imstande oder (auch nur willens) wäre. ${ }^{24}$

Scheint der emotionale wie politische und ethische Abgrund, der sich zwischen Täter und KZ-Häftlingen auftut, in der erzählten Wachwelt auch unüberwindlich, im narrativ fingierten Traum lassen sich die Opfer nicht auf Distanz halten. Physisch wie psychisch rücken sie dem Träumer auf die Pelle. Dies gilt sowohl für Hilsenraths als auch für Beyers Protagonisten: Erwähnt werden muss zunächst, dass sich auch Karnau, wie Hilsenraths Massenmörder, nach dem Krieg als Opfer ausgibt, um seine Verbrechen zu vertuschen. Hierfür erhält er einen zynischen Rat des KZ-Arztes Stumpfecker, der klingt, als sollten explizit die Körperreaktionen Miras imitiert werden, jener Überlebenden, die versuchte, den „deutschen Kloß“ im Hals loszuwerden:

Vordringlichste Aufgabe ist es nun, wie ein Opfer sprechen zu lernen [...] sprechen Sie mit niedergeschlagenen Augen, lassen Sie Pausen im Sprachfluß, als sei Ihnen Grausames widerfahren, dessen Beschreibung Sie nicht über sich bringen [...], verzerren Sie das Gesicht, stammeln Sie, lernen Sie feuchte Augen willentlich hervorzurufen [...] man wird Sie am Ende tatsächlich für ein Opfer halten, Opfer eines unbestimmten Grauens ohne Namen, für das keine Worte zu finden sind. [...] Sie müssen stottern, aussetzen, Worte verfehlen. Wir werden leider übergangsweise unter der Herrschaft gebrochener Stimmen stehen. ${ }^{25}$

Im Traum, den Marcel Beyer in Flughunde erzählt, wird allerdings aus der zunächst nur inszenierten Opferrolle ein tatsächliches körperliches Erleben der Folter. Hier erfährt der Stimmenzerstörer Karnau am eigenen Leib, wie es sich anfühlt, einem medizinischen Experiment ausgeliefert zu sein: In einem Nachkriegs-Alptraum, den er selbst in keiner Weise mit seiner eigenen Schuld in Verbindung bringt und den Marcel Beyer als literarisch ausgesprochen konstruiert und daher hochgradig unrealistisch bezeichnet hat ${ }^{26}$, befindet er sich mit einem Male in der Position des Patienten. Zwar hat er nicht, wie Mira, einen Kloß im Hals; aber mit einem Knebel im Mund zeigt sich Ohnmacht des Sprachverlusts ebenso deutlich:

Ich liege stumm, ich spüre [...] wie Fingerspitzen meinen Schädel abtasten, ich höre, wie die Haut aufreißt und ein Skalpell sich mühelos ins Fleisch gräbt [...] ich will fragen, aber auch meine Zunge spüre ich nicht, mein Gaumen, meine Lippen sind taub [...] mein Mund, als wäre er mit einer zähen Masse ausgestopft, die sich langsam mit Speichel vollsaugt, da steckt ein Knebel, mein Mund ist fest verknebelt worden. [...] ich will ein Zeichen geben, bewege Arme, Beine, Bauch, aber [...] man bemerkt meine Signale nicht, ich versuche, ein Kehlgeräusch hervorzubringen... . ${ }^{27}$

24. Zur Problematik, ob und inwiefern es aufgrund der Erzählperspektive überhaupt legitim ist, von einem „Holocaust-Roman“ zu sprechen, vgl. Simon, „Assoziation und Authentizität. Warum Marcel Beyers Flughunde auch ein Holocaust-Roman ist“, S. 126-145.

25. Beyer, Flughunde, S. 192.

26. Vgl. die Lesung und das Gespräch mit Marcel Beyer im Rahmen der Kulturprojekte des Graduiertenkollegs „Europäische Traumkulturen“ am 4. Oktober 2017 im Saarländischen Künstlerhaus Saarbrücken.

27. Beyer, Flughunde, S. 198-199; zur intertextuellen Analyse dieses Alptraums mit Blick auf Rainer Maria Rilkes Erzählung „Ur-Geräusch“ von 1919 (Rainer Maria Rilke, Gedichte und Prosa, Köln, Parkland, 1998, S. 467-473) und Herbert George Wells, The Island of Dr. Moreau, New York, Airmont, 1966 [1896], vgl. Christian Thomas, „Marcel Beyers Flughunde (1985) als Kommentar 
Gerade in dieser traumhaft erzwungenen Identifikation mit dem Opfer wird die Parallele zu Hilsenraths Nazibarbier besonders deutlich: Der Massenmörder Max Schulz, der in einem Traum plötzlich durch das Weinen der Juden an der Klagemauer angesteckt wird und selbst in Tränen ausbricht ${ }^{28}$, wird im Schlusstraum schließlich gezwungen, die Todesangst seiner ehemaligen Gefangenen vor der Erschießung unmittelbar körperlich nachzuempfinden. ${ }^{29}$ Der Traum endet mit einem schwarzen Wind, der den sterbenden Körper (mit Angstschweiß auf der Stirn, hervorquellenden Augen und aufgerissenem Mund) in den Wald der sechs Millionen ermordeten Juden trägt. ${ }^{30}$

Ein solches Traumerleben von körperlichen Grenzerfahrungen der Shoah, die sich mimetisch oder realistisch nicht darstellen lassen, soll nun in der zweiten Hälfte dieses Beitrags von der fiktionalen Inszenierung in der Gegenwartsliteratur wegführen, um die komplementäre Seite der Shoah-Literatur, gewissermaßen ihr eigentliches Zentrum zu betrachten: Texte der unmittelbaren Zeitzeugenschaft zwischen Autobiographie und Erzählung. Anhand von Traumdarstellungen der 1940er Jahre möchte ich fragen, inwiefern hier die leiblich-sinnliche Traumerfahrung genutzt wird, um die Aporie von Sprachgebot und Sprachversagen als körperliche Erfahrung auszuloten. Wie wird hier von der Unmöglichkeit des Sprechens bzw. von den Grenzen des Sagbaren erzählt?

Es geht im Folgenden um Textausschnitte aus drei Werken: Zunächst widme ich mich Traumpassagen aus der Trilogie Auschwitz et après der KZ-Überlebenden Charlotte Delbo. Sie verfasst unmittelbar nach Kriegsende ein Werk, das sich durch besondere Literarizität der erzählten leiblich-sinnlichen Erfahrungen auszeichnet sowie durch das ästhetische Ringen mit den Grenzen des Erzählbaren, das in engster Verbindung mit Fragen der Solidarität und des Erinnerns an die ermordeten Opfer steht. Sodann geht es um die Erzählung „Le songe“, die das Résistance-Mitglied Vercors bereits 1943 im Untergrund anlässlich des Berichts eines aus Oranienburg zurückgekehrten Bekannten verfasst. ${ }^{31}$ Sie gilt als die erste literarische Auseinandersetzung mit der Realität nationalsozialistischer Konzentrationslager. ${ }^{32}$ Auch hier wird der Blick auf die deportierten, inhaftierten,

zur Gegenwart der Vergangenheit“, in Inge Stephan, Alexandra Tacke (Hrsg.), NachBilder des Holocaust, Köln, Böhlau, 2007, S. 145-169, besonders S. 155-157.

28. Hilsenrath, Der Nazi Et der Friseur, S. 324-325.

29. Ibid., S. 462-465: „Ich hatte einen Wachtraum. Es kam mir vor, als läge ich wirklich im Sterben“. Im Traum erleidet er einen Herzinfarkt und soll das Herz eines Rabbiners transplantiert bekommen. „Ich höre keine Stimmen mehr. Ich sehe nichts. Es dunkelt. Es ist still. [...] Und doch sehe ich: Ich sehe, wie meine Gedanken aus der Dachschadenecke heraushüpfen, sich befreien, um die Augäpfel herumschleichen, aus den Froschaugen quellen, hervorquellen, zu schweben anfangen, im Raum herumschweben, an der Zimmerdecke hocken, mich anstarren, mir etwas zuflüstern“ (S. 464).

30. Ibid., S. 465.

31. Vgl. hierzu Jean-Paul Dufiet, „Novembre 1943, ,Le Songe“ de Vercors: la littérature et le camp de concentration nazi“, Roman 20-50 28, 1999, S. 115-127, hier S. 115.

32. Zum Entstehungskontext und zur Rezeption vgl. ausführlicher Christiane Solte-Gresser, „,Le Songe“ (Vercors, d.i. Jean Bruller)“, Online-Lexikon Traumkultur, 11. August 2016, http:// traumkulturen.uni-saarland.de/Lexikon-Traumkultur/index.php/“Le_Songe“_(Vercors,_d.i._ Jean_Bruller) [Stand: 17. Juni 2018]. 
gequälten und ermordeten Opfer, deren Körperlichkeit radikal ins Zentrum gerückt wird, zur entscheidenden erzähltechnischen Konstruktion. Und auch hier ist es eben der Modus des Traums, der eine Identifızierung mit den Vernichteten ermöglicht. Allerdings wird diese Identifizierung nicht erzwungen, wie dies in allen anderen Beispielen der Fall ist, sondern als bewusste Entscheidung von Träumer und angesprochenen Lesern vorgeführt. Abschließend soll noch ein kurzer Blick auf Les Bagages de sable von Anna Langfus geworfen werden, eine derjenigen Shoah-Überlebenden, die ihre autobiographischen Erfahrungen auf ausdrücklich fiktionale Weise verarbeiten. ${ }^{33}$ Denn die Traumepisoden dieses Romans stehen in ganz besonders enger Verbindung mit den seelischkörperlichen Wahrnehmungen der Hauptfigur. Mit Anna Langfus schlagen wir daher den Bogen wieder zurück zum Anfang, nämlich zum Problem der ,Schuld“ der Überlebenden, die im Traumerleben ihren extremen körperlichen Ausdruck findet. Insgesamt bewegen wir uns auf diese Weise von der Außensicht der Täter zunehmend in die literarisch inszenierten Körpererfahrungen der Deportierten innerhalb der Konzentrationslager hinein, um uns abschließend nochmals der Körperwahrnehmung der traumatisierten Überlebenden zuzuwenden.

Auch wenn Marcel Beyer mit Flughunde, einen hochgradig fiktionalen Roman vorlegt, betont der Autor selbst die zahlreichen verifizierbaren Daten und Fakten des nationalsozialistischen Kontextes. ${ }^{34}$ So ist der fiktionale KZ-Arzt Stumpfecker, mit dem Karnau zusammen seine Stimmexperimente durchführt, in offenkundiger Weise an Hitlers Leibarzt Ludwig Stumpfegger angelehnt, der im KZ Ravensbrück Menschenversuche an polnischen Frauen durchgeführt und für seine Habilitationsschrift genutzt hatte.

Charlotte Delbo, die im Anschluss an ihre Auschwitz-Gefangenschaft nach Ravensbrück deportiert wird, deutet in Une connaissance inutile, dem zweiten Band ihrer Autobiographie, eben jene Menschenexperimente an, denen ihre Mitgefangenen ausgesetzt sind. Ohne Weiteres hätte sie also selbst eines von Stumpfeggers tatsächlichen Opfern sein können. In ihrem Werk ergreift sie ausdrücklich und sehr programmatisch die Stimme für diejenigen, deren Sprache ausgelöscht, deren Namen vergessen, deren Körper nicht begraben und nicht betrauert wurden. ${ }^{35}$ Dies zeigt sich etwa daran, dass sie ganze Kapitel über einzelne Frauen schreibt, die sie hat sterben sehen, dass die Eigennamen der erinnerten Opfer geradezu leitmotivartig wiederholt werden, dass sie GedenkGedichte bestimmten getöteten Freundinnen widmet; vor allem aber auch daran, dass ein kollektives „nous“ das sprechende Ich überlagert oder sich ganze Textpassagen an ein „vous“ richten - als über den Tod fortdauernder Dialog mit den Toten. Nicht zuletzt ist die Trilogie damit auch eine verzweifelte

33. Zur besonderen Position von Langfus auf dem Feld der Shoah-Literatur vgl. Joe Friedemann, Langages du désastre, Saint Genouph, Nizet, 2007, S. 107-141, hier S. 112.

34. Vor allem die historisch identifizierten Täter dienen - auch über Goebbels hinaus - als Vorbilder für Beyers Figurengestaltung.

35. Zur Besonderheit der Erzählstimme vgl. ausführlicher Silke Segler-Meßner, „Le genre, le récit et le corps. „Aucun de nous ne reviendra' de Charlotte Delbo et ,L'Espèce humaine“ de Robert Antelme“, Romanische Studien 2, 2015, S. 81-104, hier insbesondere S. 85, 87 und 93. 
Hommage an die Solidarität jener Mitgefangenen, deren Tod - zumindest in der Wahrnehmung des erzählenden Ich - zum eigenen Überleben beigetragen hat. Für unseren Zusammenhang sind drei Besonderheiten des Werkes entscheidend, die hier nur sehr schlaglichtartig ausgeführt werden können ${ }^{36}$ : Die schockierende Deutlichkeit, mit der die geschundenen Körper inszeniert werden, die radikale Fokussierung auf eine weibliche Körperperspektive, mit der das Werk Delbos deutlich aus dem Feld autobiographischer Shoah-Literatur heraussticht ${ }^{37}$, und die ausgesprochen ästhetische, traumhaft-poetische Schreibweise, mit der das Erlebte in eine autobiographische Erzählung überführt wird. ${ }^{38}$ Wie untrennbar die körperliche Zerstörung ganz konkret mit dem Verlust von Stimme und Sprache einhergeht, zeigt sich durchgängig in allen drei Bänden der Trilogie; beispielhaft etwa an folgender Passage:

Soif à en perdre la raison, soif à ne plus pouvoir manger, parce que je n'avais pas de salive dans la bouche, soif à ne plus pouvoir parler, parce qu'on ne peut plus parler quand on n'a pas de salive dans la bouche. Mes lèvres étaient déchirées, mes gencives gonflées et ma langue un bout de bois. Mes gencives gonflées et ma langue gonflée m'empêchaient de fermer la bouche, et je gardais la bouche ouverte comme une égarée, les pupilles dilatées, les yeux hagards. ${ }^{39}$

Eines der zentralen Motive des Delbo'schen Schreibens ist daher der menschliche Schrei, der - als sei hier das sprachliche Bild Kofmans in die konkrete Körpersprache übersetzt - sich lautlos vollzieht, der im Halse stecken bleibt. Als Tausende von Frauen in Auschwitz mit ansehen müssen, wie eine fliehende Mitgefangene durch einen Wachhund der SS zerrissen wird, heißt es:

Comme dans un rêve. La femme crie. Un cri arraché. Un seul cri qui déchire l'immobilité de la plaine. Nous ne savons pas si le cri vient d'elle ou de nous, de sa gorge crevée ou de la nôtre. Je sens les crocs du chien à ma gorge. Je crie. Je hurle. Aucun son ne sort de moi. Le silence du rêve. [...] Il y a eu un râle qui n'a pas pu sortir de sa gorge [...]. On aurait dit qu'on l'avait étranglée. ${ }^{40}$

36. Vgl. hierzu ausführlicher Solte-Gresser, „Das Sterben der Anderen“.

37. Vgl. hierzu Elizabeth Roberts Baer, Myrna Goldenberg, Experience and Expression: Women, the Nazis, and the Holocaust, Detroit, Wayne State UP, 2003 und Caroline Moorehead, A Train in Winter: An Extraordinary Story of Women, Friendship, and Resistance in Occupied France, New York, Harper Collins Publishers, 2011.

38. Vgl. Judith Klein, Literatur und Genozid: Darstellungen der nationalsozialistischen Massenvernichtung in der französischen Literatur, Wien, Böhlau, 1992, S. 78-95. Eine besondere Rolle kommt dabei jenen Menschen auf der Grenze zwischen Leben und Tod zu, die bei Levi, Frankl und vielen anderen bekanntlich Muselmänner genannt werden, und die Charlotte Delbo neben der weiblichen Formulierung „les musulmanes“ (Charlotte Delbo, Mesure de nos jours, Bd. 3 der Trilogie Auschwitz et après, Paris, Minuit, 1971, S. 9 und S. 142-146) auch „mannequins“, „squelettes“, aber auch „spectres“ oder „revenants“ nennt (Charlotte Delbo, Une connaissance inutile, Bd. 2 der Trilogie Auschwitz et après, Paris, Minuit, 1970, S. 187188). Insofern ist es konsequent, wenn Beyer in Flughunde aus der Sicht Karnaus die Opfer als Schemen oder schwarze Schatten bezeichnet (Beyer, Flughunde, S. 11).

39. Delbo, Une connaissance inutile, S. 41-42.

40. Charlotte Delbo, Aucun de nous ne reviendra, Bd. 1 der Trilogie Auschwitz et après, Paris, Minuit, 1970, S. 48. 
Die Erinnerung, wie zusammengepferchte Frauen auf einem Lastwagen vorbeifahren, beschreibt Delbo folgendermaßen:

Les femmes crient vers nous [...] sans qu'aucun son nous parvienne. Leurs bouches crient, leurs bras tendus vers nous crient et tout d'elles. Chaque corps est un cri. [...] Chacune est un cri matérialisé, un hurlement - qu'on n'entend pas. [...] Elles hurlaient parce qu'elles savaient, mais les cordes vocales s'étaient brisées dans leur gorges. ${ }^{41}$

Ebenfalls im ersten Band der Trilogie, Aucun de nous ne reviendra, findet sich ein Alptraum, in dem sich die tagtägliche Zwangsarbeit in den Sümpfen um Auschwitz mit den nächtlichen Halluzinationen im Halbschlaf zum Eindruck vermischt, im Schlamm zu ersticken. Diese Traumerfahrung verweist in geradezu verblüffender Weise motivisch, thematisch und stilistisch auf die Traumerzählung von Vercors, die uns gleich im Anschluss beschäftigen wird.

[...] être étranglées par un tentacule qui s'enroulait autour du cou, serrait les vertèbres, les serrait à les craquer, les vertèbres, la trachée, l'œsophage, le larynx, le pharynx et tous les conduits qu'il y a dans le cou [...]. Il fallait libérer la gorge [...] : Ce ne sont pas les pieuvres, c'est la boue [...] [qui] entre dans les yeux, dans le nez, dans la bouche, suffoque [...]; notre trague est remplie d'yeux et de dents, d'yeux qui luisent, de dents qui ricanent [...] et tous ces yeux et toutes ces dents flamboient et vocifèrent, dardant, mordant de toutes parts et hurlant: schneller, schneller, weiter, weiter [...] et quand nous donnons des coup de poing dans ces gueules toutes en dents et en yeux, les poings ne rencontrent que taies molles, éponges pourries. ${ }^{42}$

Von diesem Alptraum einer Überlebenden im Konzentrationslager ist es nur ein kleiner Schritt zu einem Alptraum über ein Konzentrationslager, den die Erzählstimme in Vercors „Le songe“ zu träumen vorgibt: In seinem knapp sechsseitigen Traumbericht schildert das erzählende Ich, wie es angsterfüllt durch eine sumpfige, geisterhafte Landschaft irrt. Ein Mann versucht, Kontakt aufzunehmen, doch die Kommunikation scheitert; die beiden Gestalten können sich nicht verständigen. ${ }^{43}$ Während der Erzähler erschrocken zurückweicht, nimmt er weitere Gestalten wahr, die nicht minder entstellt sind: Abgemagert, verstümmelt und monströs verzerrt, bewegen sie sich gespensterhaft durch die Sümpfe. Auch ihnen gelingt es nicht, sich sprachlich zu artikulieren. Sie krümmen und winden sich lediglich, als bäumte sich der Organismus auf und brächte sich durch „rote Schreie“ und „weißliches Wimmern“ zu Gehör. ${ }^{44}$ Auf diejenigen Gestalten, die zusammenbrechen und im Morast liegenbleiben, prügeln schwarz gekleidete Männer mit Knüppeln ein. Seinen Höhepunkt erreicht der Bericht, als das Ich selbst in körperlichen Kontakt mit einem Glied aus den Reihen des Todesmarsches gerät: Er tritt auf eine Hand, die sich wie eine weiche Krake anfühlt, während der Sterbende seinen leeren Blick auf ihn richtet.

41. Ibid., S. 55-57.

42. Ibid., S. 86-88.

43. „Il ouvrit la bouche et je vis l'horrible langue tordue, racornie, noire et déchirée, qui s'enroulait comme un escargot cuit“. Vercors (Jean Bruller), „Le songe“, in ders., Le Silence de la mer et autres auvres, Paris, Omnibus, 2002, S. 177-186, hier S. 181.

44. „[C]omme si l'organisme se fût révolté, eût voulu protester, se faire entendre par ces cris rouges ou ces gémissements blanchâtres“. Ibid., S. 182. 
An dieser Stelle gibt der Erzähler seinen schockartigen Erkenntnisprozess wieder: Während er Verwesungsgeruch, aschefarbenen Rauch und tätowierte Nummern in der Haut der Traumgestalten wahrnimmt, erkennt er endlos aufgereihte Baracken und versteht die Funktion des Kamins am Horizont. Beendet wird die Erzählung mit dem Blick jenes lächelnden Mannes, der dem Träumer bereits zu Beginn erschienen war. Dieser sendet ihm sterbend mit seinem Lächeln ein letztes brüderliches Zeichen ${ }^{45}$, das er mit der Hoffnung verbindet, nicht vergessen zu werden.

Die Erzählung Vercors' wird gerahmt von einer programmatischen Einbeziehung des Lesers in das Geschehen. In besonderen Nächten, heißt es, habe man im Traum Anteil an etwas, das nicht nur jenseits des Verstandes, sondern auch jenseits der Imagination und damit jenseits des Beschreibbaren liegt, die sich aber unauslöschlich in die leibliche Wahrnehmung des Träumers eingegraben hat. ${ }^{46}$ Der Träumer, der zu Beginn mitleidig oder entsetzt die erträumten Figuren beobachtet, lässt sich am Schluss nicht mehr unterscheiden von den leidenden und entsetzlichen Gestalten, die ihm im Traum begegnen und zulächeln. ${ }^{47}$

Besonders ausgeprägte Merkmale des Textes sind die groteske Verzerrung und monströse Körperlichkeit der Traumgestalten. Ohne Kenntnis der historischen Fakten müssen sie dem Leser wie eine karikaturhafte Überzeichnung des Geschehens erscheinen. Einzig aus der Perspektive des (späteren) Wissens über den ,Muselmann“ im Lager ${ }^{48}$, lässt sich erkennen, dass es sich bei der Beschreibung der KZ-Häftlinge als wandelnde Skelette nicht um eine Übertreibung handelt. Den größten Raum des Textes nehmen die körperlichen Beschreibungen der Geschundenen ein, deren Grad der Entstellung und Entmenschlichung sich an den Grenzen des Erträglichen bewegt und deren Beschreibung zwischen

45. Ibid., S. 186.

46. Denn was für das erzählende Ich nach dem Erwachen zurückbleibt, sind vor allem die im Traum erlebten sinnlichen Eindrücke und die Erkenntnis, dass die Grenzen zwischen Traum und Wachwelt unwiderruflich eingerissen sind. Zur Traumkonzeption im historisch-kulturellen Kontext vgl. ausführlicher Christiane Solte-Gresser, „Traumdiskurse des beschädigten Selbst. Traumwissen in Konzentrationslagerträumen vor 1950“, in Hendrik Schlieper, Leonie Sülwolto, Jörn Steigerwald (Hrsg.), Komparatistik heute. Aktuelle Positionen der Vergleichenden Literaturwissenschaft, Paderborn, Fink (im Druck).

47. „Maintenant j'étais un de ces hommes. Je ne le suis pas devenu. Je l'étais. Depuis toujours. Je n'étais plus ce spectateur [...]. J'étais seulement un de ces hommes-là“. Vercors, „Le songe“, S. 186.

48. Z.B. bei Viktor Emil Frankl, Trotzdem Ja zum Leben sagen. Ein Psychologe erlebt das Konzentrationslager, München, dtv, 1988 [1947]; Primo Levi, Se questo è un uomo, Torino, Einaudi, 2005 [1958]; Antelme, L'Espèce humaine, S. 98, aber auch Delbo, Mesure de nos jours, S. 142-146. Aus theoretisch-philosophischer Perspektive werden sich für unseren Zusammenhang die Reflexionen von Giorgio Agamben als bedeutsam erweisen: Giorgio Agamben, Quel che resta di Auschwitz. L'archivio e il testimone, Torino, Bollati Boringheri, 1998; dt.: Was von Auschwitz bleibt. Das Archiv und der Zeuge (Homo sacer III), Frankfurt a. M., Suhrkamp, 2003, vor allem S. 36-75. Dezidierter auf literarische Texte bezieht sich Mona Körte, „Stummer Zeuge. Der ,Muselmann“ in Erinnerung und Erzählung“, in Silke Segler-Meßner, Monika Neuhofer, Peter Kuon (Hrsg.), Vom Zeugnis zur Fiktion. Repräsentation von Lagerwirklichkeit und Shoah in der französischen Literatur nach 1945, Frankfurt a. M., 2006, S. 97-110. 
naturalistischen und fantastischen Erzählverfahren changiert. Beispielsweise wird nicht nur die Zunge des KZ-Häftlings mit einer zusammengerollten Schnecke verglichen; die Gesichter der Gestalten gleichen bleichen Endivien, ihre Haut wirkt wie verdorbene Milch, Augenlider werden zu abgenutztem Seidenpapier, der Mund ist zwischen zwei glühenden Klammern verschwunden. ${ }^{49}$

Mit dem verzweifelten Bemühen der Traumgestalt, trotz verstümmelter Zunge zu sprechen und dem Hinweis auf einen feurig verklammerten Mund eröffnet der Traum zugleich eine poetologische Dimension: Es geht innerhalb der Beschreibung des Geschehens auch um die grundsätzliche Frage, wie sich die „unerklärliche Katastrophe“ ${ }^{50}$ beschreiben lässt, ja ob sie überhaupt darstellbar ist, ohne sie durch Pathos oder Groteske unglaubwürdig zu machen. ${ }^{51}$ Denn die versengte Zunge verhindert die Kommunikation der Erfahrung, weil sie sich verkrampft, einrollt und verhärtet. Aber auch die wie zerfetzte Zungen vom Himmel herabhängenden Nebelschwaden, die als Stoffbahnen ${ }^{52}$ beschrieben werden, sind im Text direkt mit dem Problem des Mitteilens verbunden. Die Formulierung „des langues déchiquetées“ ist sicherlich nicht zufällig gewählt. Denn der mehrfach verwendete Begriff „langue“ meint ja sowohl das Sprechorgan als auch die Sprache selbst. Nadja Lux hat darüber hinaus gezeigt, dass sich die Versuche, das Erlebte zu kommunizieren, hier vor allem nonverbal, d.h. durch körperliche Gesten und Mimik, vollziehen. ${ }^{53}$ Das literarische Verfahren des Autors bewegt sich zwischen den beiden Extremen der mimetischen Repräsentation und der traumhaften Verfremdung des Geschehens. Im Bewusstsein der Unmöglichkeit des treffenden Ausdrucks wird gleichwohl eine möglichst weitgehende sprachliche Annäherung versucht. Vercors' Traumerzählung wäre damit eine ästhetische Antwort „auf das Dilemma zwischen dem Unvermögen der Darstellung und dem gleichzeitig als moralische Pflicht empfundenen Zeugnisgebot““. ${ }^{54}$

Kehren wir abschließend von der geträumten Körpererfahrung im Konzentrationslager zurück zu den körperlichen Symptomen der traumatisierten Überlebenden. Bei Vercors war es die physische und psychische Identifikation zwischen Außenstehendem und KZ-Häftlingen im Traum, mit der das ausgelöschte Sprachvermögen nach außen getragen, erzählend vermittelt werden soll. Delbo hat die eigene körperliche Zerstörung als Erfahrung eines kollektiven Wir erzählt. Gerade aufgrund der poetisch eindringlichen Schreibweise der Gewalt hinterlässt sie, einer Traumerfahrung gleich, tiefe Spuren auch bei den Leserinnen und

49. Mitunter bildet die Krankheitsmetaphorik die einzig bleibende Verbindung zum menschlichen Körper, vgl. hierzu Nadja Lux, „Alptraum: Deutschland“, Traumversionen und Traumvisionen vom „Dritten Reich“, Freiburg, Rombach, 2008, S. 347.

50. „[L]a catastrophe inexplicable“. Vercors, „Le songe“, S. 182.

51. Vgl. Frédéric Canovas, „Ce que tout lecteur doit savoir: Vercors, le rêve et l'écriture de la mort“, in Georges Cesbron, Gérard Jacquin, Vercors et son œuvre (Actes du colloque, Université d'Angers, Mai 1995), Paris, L'Harmattan, 1999, S. 417-436, hier S. 423.

52. Vercors, „Le songe“, S. 180.

53. Lux, „Alptraum: Deutschland“, S. 340-341.

54. Vgl. ibid., S. 358. Die Verfasserin bezeichnet dieses Verfahren hier überzeugend als asymptotisches Erzählen, eine fortwährende Annäherung an eine Erfahrung, die als solche zu kommunizieren ein unerreichbares Unterfangen bleibt. 
Lesern ihrer Trilogie. Uns allerdings gelingt es, deutlich zwischen ästhetischer Immersion, traumhaftem Erleben und Wachwirklichkeit zu unterscheiden. Diese Differenzierung ist Delbo, wie so vielen anderen Überlebenden ${ }^{55}$, nicht mehr möglich. Und selbst die Alpträume der Überlebenden, von denen sie später berichtet, ähneln sich untereinander und handeln noch von Schreien, die die schmerzende Brust nicht verlassen können. ${ }^{56}$ Es sind dies eben die körperlichen Reaktionen, die Hilsenraths und Beyers Täter so schamlos instrumentalisiert hatten, um der eigenen Schuld nicht ins Auge blicken zu müssen. Solche Symptome der sprachlichen Ohnmacht, des stimmlichen Versagens und des Erstickens an Worten finden sich besonders ausgeprägt auch bei Anna Langfus. Tragischerweise ist es hier ebenfalls das Gefühl von Schuld ${ }^{57}$ - aber eben, wie bei Delbo, der Schuld des eigenen Überlebens auf Kosten der anderen -, das sich tief in das Körpererleben eingraben hat: Es beschneidet die Stimme und scheint das Ich zu erdrosseln.

Die Ich-Erzählerin aus Les Bagages de sable, eine allein aus dem Konzentrationslager zurückgekehrte lebensmüde junge Frau, kämpft mit körperlichen Beschwerden wie Erstickungsanfällen, Atemlosigkeit, damit, dass ihr die Worte im Halse stecken bleiben. In auffälliger Weise erinnern die Motive, die Anna Langfus verwendet, an unsere anderen Beispiele: Der Hals ist ausgetrocknet, wenn Maria mit ihren Erinnerungen konfrontiert wird. Das Essen breitet sich auf unangenehme Weise in ihrem Mund aus. ${ }^{58}$ Ein bestimmtes tröstliches Wort soll die Kugel, die den Hals verstopft, zum Schmelzen bringen; auch sie hat es verlernt, zu weinen. ${ }^{59}$ Und der Schrei der Gefolterten, den die Überlebenden „auf immer in ihrem Körper tragen werden“"60, ist derselbe, den das Ich unvermittelt auf offener Straße ausstößt - auch wenn er von niemandem gehört wird. ${ }^{61}$

Das Thema der versagenden Sprache durchzieht den gesamten Text ebenso wie zahlreiche Träume, Halluzinationen und traumatische flash-backs. ${ }^{62}$ Wenn

55. Vgl. das Träumen als vorherrschendes Erzählverfahren im autobiographischen Schreiben von Jorge Semprún, aber auch den berühmten Schlusstraum aus Primo Levis La tregua und die Verwirrung zwischen Träumen und Wachen in Langfus' Bagages de sable, vgl. hierzu Christiane Solte-Gresser, „Cauchemars concentrationnaires. Écrire l'expérience historique à travers le rêve: Approches d'une poétique onirique (1953-1963)“, in Bernard Dieterle, Manfred Engel (Hrsg.), Historizing the Dream/Le Rêve du point de vue historique, Würzburg, Königshausen \& Neumann, 2018, S. 291-309.

56. Delbo, Mesure de nos jours, S. 194-199, hier besonders S. 198.

57. Vgl. Jean-Paul Dufiet, „Le sujet et l'expérience de la Shoah chez Anna Langfus“, in Pierre Glaudes (Hrsg.), L’Expérience des limites dans les récits de guerre (1914-1945), Genève, Slatkine, 2001, S. 161-174, hier S. 173.

58. Beide Beispiele Anna Langfus, Les Bagages de sable, Paris, Gallimard, 1962, S. 17.

59. Ibid., S. 14 und S. 24.

60. Ibid., S. 40.

61. Ibid., S. 20.

62. Weder dem väterlichen Freund, der die orientierungslos Umherirrende aufgabelt, noch einem zufällig getroffenen KZ-Überlebenden, mit dem sie offensichtlich zahlreiche Erlebnisse teilt, ohne sie jedoch explizit kommunizieren zu können, noch jenen Verwandten, die das Mädchen zu sich aufnehmen, in dem sie nur das Opfer sehen und es damit ein weiteres Mal entfremden, 
die Überlebende spricht - jedenfalls in einer Weise spricht, die sich jenseits der leeren Worte und des Schwindels vollzieht, über den Sarah Kofman nachdenkt -, so geschieht dies allenfalls im Traum: Hier trifft sie regelmäßig auf die ermordeten Angehörigen, die der Protagonistin, vielsagend schweigend oder in harschen Worten, ihr Überleben vorwerfen. Sehen wir uns abschließend ein Beispiel an, das in seiner dichten Symbolik all die unterschiedlichen zuvor präsentierten Traumepisoden gewissermaßen zusammenzufassen scheint. ${ }^{63}$

Im Übergang vom Wachzustand in den Schlaf nimmt die erschöpfte Erzählerin eigenartige Körpersymptome wahr: Der Klang aus dem Bett fallender Schuhe dröhnt schmerzlich im Kopf, der Leib zittert trotz der Sommerhitze, im Kiefer haben sich zu viele Zähne ausgebreitet (die sich verselbständigenden Zähne erinnern in verblüffender Weise an den Alptraum Delbos). Ein Mann tritt an ihr Bett und versucht vergeblich, Kontakt aufzunehmen. An seinem Rücken hängt ein schwerer Stein, dessen Gewicht auch sie selbst nach unten zieht. Denn dieser Stein ist mit einem Seil an ihrem eigenen Hals befestigt. Als die Träumerin dieses Seil mit einem Messer durchtrennen will, um sich selbst und denjenigen zu befreien, der plötzlich Ähnlichkeit mit dem eigenen Vater aufweist, muss sie feststellen, dass es sich um eine lebendige, pulsierende Vene handelt, die beide untrennbar miteinander verbindet. Die Toten nehmen ihr die Luft zum Atmen; doch ablösen kann sie sich von ihnen nur, wenn sie sich zugleich die eigene Lebensader durchschneidet. $\mathrm{Zu}$ tragen hat die Träumerin die Last einer Vergangenheit, die mit einem Nabelschnur ähnlichen Seil an den Körper fixiert bleibt und damit die Worte im Hals regelrecht abschnürt. ${ }^{64}$

kann sie sich mitteilen. Zu Langfus' Sprache des Irrealen vgl. Madeleine Cottenet-Hage, „Anna Langfus et les risques de la mémoire“, in Vincent Engel (Hrsg.), La littérature des camps: la quête d'une parole juste, entre silence et bavardage, Les lettres romanes, $\mathrm{n}^{\circ}$ spécial hors série, 1995, S. 25-39.

63. Vgl. hierzu auch ihre eigenen Aussagen vom März 1961 in L'Arche; zitiert nach: Judith Kauffmann, „Pour (re-)lire Anna Langfus. Survire/Résister. Et Après...“, in Georges Bensoussan, Myriam Ruszniewski-Dahan (dir.), La Shoah dans la littérature française, Revue d'histoire de la Shoah 176, 2003, S. 100-116, hier S. 115. Unmittelbar im Anschluss daran (S. 116) findet sich auch die für unseren Zusammenhang wichtige Aussage: „L'horreur a sa propre langue et la voix d'un homme sera toujours trop faible pour la rendre. Souvent, le cri ne peut être traduit que par le silence“. Sie erzähle in ihren Texten viel vom Schweigen und der Stille, denn die Stimme breche vor lauter Schreien angesichts der Frage, wie man als Außenstehender in literarischen Texten die Opfer hörbar machen kann. Andernorts ist der Essay bezeichnenderweise publiziert unter dem Titel „Un cri ne s’imprime pas“ (Lévy, „La guerre“, S. 52- 60, hier S. 59).

64. Der subjektive Eindruck schuldhafter Verstrickung der Überlebenden wird in Szene gesetzt, indem die Träumende nicht nur selbst an der Belastung zu ersticken droht, sondern zugleich untrennbar an die deportierte Vater-Figur gebunden bleibt. Der Traum endet mit einer demütigenden Prüfungssituation, die das Thema der Unaussprechlichkeit nochmals ganz konkret in eine gescheiterte Kommunikationssituation überträgt: Die Träumende soll in der Schule die Namen sämtlicher Konzentrationslager aufsagen. Sie jedoch hat das Bedürfnis, stattdessen „tous les détails“ auszusprechen. Diese Erfahrungen jedoch bekommen keinen Raum; stattdessen beißt sich die Träumerin auf die Zunge, weil sie gezwungen wird, den Namen eines bestimmten geflohenen Mithäftlings auszusprechen. Vgl. hierzu Christaine Solte-Gresser, „Les Bagages de sable“ (Anna Langfus)“, Online-Lexikon Traumkultur, 5. August 2016, http://traumkulturen. uni-saarland.de/Lexikon-Traumkultur/index.php?title=\%22Les_bagages_de_sable\%22_(Anna_ Langfus,_geb._Szternfinkiel)Etoldid=870 [Stand: 17. Juni 2018]. 
Nach Giorgio Agamben besteht die Aporie der Shoah darin, „in doppeltem Sinne ein Ereignis ohne Zeugen“ zu sein. Ihm zufolge ist es „ebenso unmöglich, aus dem Inneren des Todes Zeugnis abzulegen - es gibt keine Stimme für das Verschwinden der Stimme -, wie von außen her - denn der outsider ist per definitionem vom Ereignis ausgeschlossen“. ${ }^{65}$ Allerdings heißt, im Paradigma der Unsagbarkeit zu verharren, für Agamben auch, „zu seinem [Auschwitz'] Ruhm beizutragen“ und damit unwillentlich die Geste der Nationalsozialisten $\mathrm{zu}$ wiederholen. ${ }^{66}$ Seine Konsequenz lautet daher, „den Blick fest auf das Unaussprechliche zu heften. Auch auf die Gefahr hin, entdecken zu müssen, dass wir das, was das Böse von sich weiß, leicht auch in uns finden“. ${ }^{67}$ Einer solchen Banalität des Bösen ${ }^{68}$ ins Auge zu blicken, dazu haben uns die Romane von Beyer und Hilsenrath durch ihre radikale Innenperspektive gezwungen. Aber besonders die autobiographischen Texte, die ich einer vergleichenden Lektüre im Hinblick auf körperliche Traumerfahrungen der Shoah unterzogen habe, kommen Agambens Forderung nach, die „Unmöglichkeit zu sprechen zum Sprechen zu bringen“" ${ }^{69}$. Damit lässt sich das Körper-Schreiben von Anna Langfus, Charlotte Delbo und Vercors selbst als eine ästhetisch ausgesprochen konsequente Auseinandersetzung mit dem Topos der Unsagbarkeit verstehen. Indem die (autobiographischen oder fremden) Erfahrungen der gewaltsamen Zerstörung von Körpergrenzen hier bis in die letzten Verästelungen nachvollzogen werden, setzen die Erzählungen das Versagen der Sprache und die Undarstellbarkeit der erfahrenen Wirklichkeit körperlich in Szene; und zwar als existenzielle Grenzerfahrung im wörtlichen Sinne: Rachen, Kehlkopf, Zunge, Stimmbänder und Lunge erweisen sich als die Organe, mittels derer eine Körpersprache des Verstummens inszeniert wird. So werden die somatischen Grenzen sichtbar, an denen sich die traumatische Erfahrung in das Körpergedächtnis einschreibt und das Erzählen selbst an seine Grenze führt.

65. Agamben, Was von Auschwitz bleibt, S. 31.

66. Ibid., S. 137.

67. Ibid., S. 29. Und dies tut er bekanntlich in intensiver und konsequenter Auseinandersetzung mit einem weiteren wichtigen Traum-Autor der Shoah, nämlich Primo Levi.

68. Vgl. Hannah Arendt, Eichmann in Jerusalem. Ein Bericht von der Banalität des Bösen, München, Piper, 2006 [1963].

69. Agamben, Was von Auschwitz bleibt, S. 143. 
